\title{
The Way to an Ideal Matter-free Zernike and Hilbert TEM Phase Plate: Anamorphotic Design and First Experimental Verification in Isotropic Optics
}

\author{
N. Frindt, ${ }^{*}$ K. Schultheiss, ${ }^{* *}$ B. Gamm, ${ }^{* *}$ M. Dries, ${ }^{* *}$ J. Zach, ${ }^{* * *}$ D. Gerthsen, ${ }^{* *}$ and R.R. \\ Schröder* \\ * Universität Heidelberg, CellNetworks, BioQuant, D-69120 Heidelberg, Germany \\ ** Laboratorium für Elektronenmikroskopie, Karlsruher Institut für Technologie, D-76128 \\ Karlsruhe, Germany \\ *** CEOS GmbH, Englerstr. 28, D-69126 Heidelberg, Germany
}

Physical phase plates of different types and experimental realizations have been proposed and tested for optimizing phase contrast in the TEM [1-4]. Common to most of the schemes is an unwanted obstructing object - such as carbon film, drift tube electrodes, centrosymmetric electrostatic einzel lenses or magnetic wires - in one of the conjugate diffraction planes of the microscope. Theoretical work has shown [5] that for realization of the full potential of phase plate microscopy it will be mandatory to minimize or - even better - to eliminate any blocking matter related to a physical phase plate.

Recently we proposed a matter-free slit-like electrostatic phase plate [6], which needs special electron optics to form a highly anamorphotic image of the diffraction plane (cf Fig. 1). While such electron optics is still under development [7] realistic phase plate designs derived from finite element modelling have to be characterized for their expected phase shifting properties. Fig. 2 shows a typical distribution of the calculated potential in 3D and its resulting projected phase shift. The optimized design of the electrostatic slit phase plate, which we present here, is therefore setting constraints for the necessary anamorphotic ratio of the distorted diffraction plane.

In addition we show specific object contrast properties during the phase plate alignment steps. This illustrates that object contrast as image read-out may be used to align anamorphotic slit phase plate and optics. It is particularly helpful to combine Zernike- and Hilbert-type electrostatic potentials to implement automatic alignment procedures. Fig. 3 illustrates this in a simple electrode case such as the Zach phase plate [8].

As first experimental tests of these ideas we show examples of phase contrast images obtained when using the Zach phase plate in conventional round optics [9]. In principle we can experimentally simulate the use of the slit phase plate in Zernike-mode by using two Zach phase plates in opposition. Experimental verification of such geometries is under way.

References

[1] R. Danev et al., Ultramicroscopy 88 (2001) 243.

[2] K. Schultheiß et al., Rev. Sci. Instrum. 77 (2006) 033701.

[3] R. Cambie et al., Ultramicroscopy 107 (2007) 329.

[4] K. Nagayama, Proc. M\&M 2007, Ft. Lauderdale, USA.

[5] B. Gamm et al., Ultramicroscopy, DOI: 10.1016/j.ultramic.2010.02.006.

[6] R.R. Schröder et al., Proc. M\&M 2007, Ft. Lauderdale, USA.

[7] H. Rose, contribution to this conference. 
[8] J. Zach, patent pending: WO/2008/061603, PCT/EP2007/009289.

[9] K. Schultheiß et al., contribution to this conference.

[10] The project is funded by the German Research Foundation (Deutsche Forschungsgemeinschaft) under Ge 841/16 and Sch 424/11.

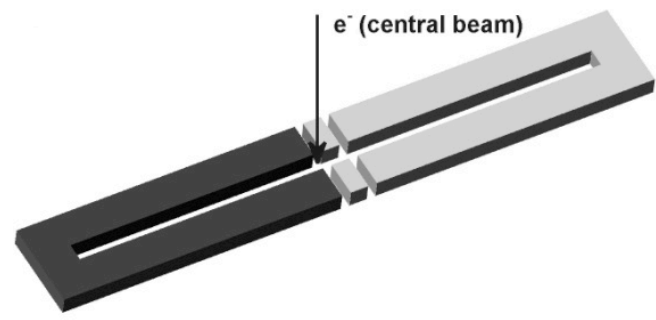

anamorphotic Hilbert phase plate

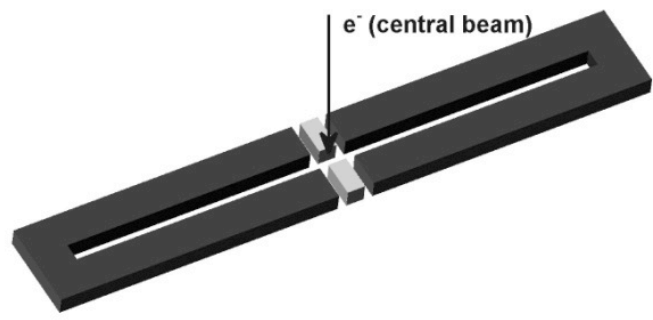

anamorphotic Zernike phase plate

FIG. 1. Schematic design of the inner four electrodes and their potential for Hilbert-type (left) and Zernike-type (right) phase contrast. The upper and lower grounding electrodes are not shown, $\mathrm{cf}$. [x].
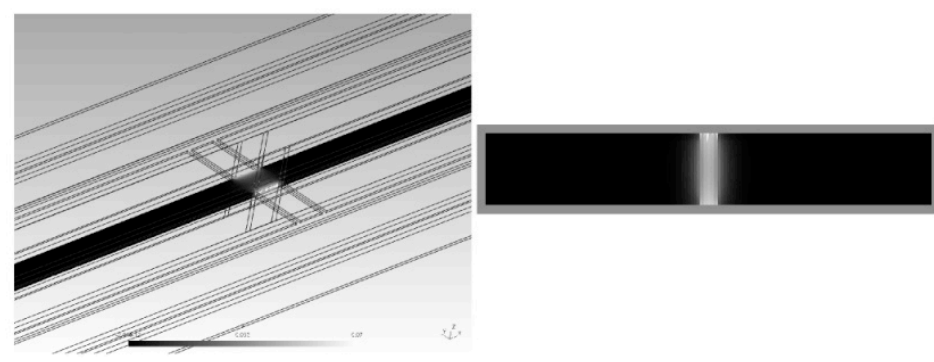

FIG. 2. (upper panel) Finite element modelling of the potential distribution around the slit phase plate as Zernike-type phase plate: (left) slice through 3D distribution of potential, (middle) integrated potential (phase shift) projected along a proposed orthogonal electron ray path, (right) resulting phase shift profile along slit for a 1:100 anamorphotic xy-ratio, cf. [7].

FIG. 3. (right panel) Contrast read-out for misaligned and aligned phase plate in the case of one Zach phase plate [8] (upper row: images, lower row: power spectra). In the simulations the distance of the unscattered beam from the electrode is $\mathrm{y}=300 \mathrm{~nm}$, the $\mathrm{x}$-direction misalignment is $1.6 \mu \mathrm{m}$ (left), while (right) illustrates a perfectly aligned phase plate.

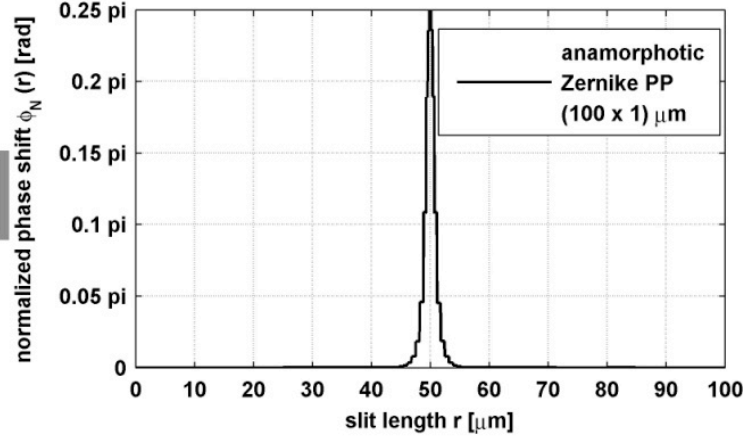

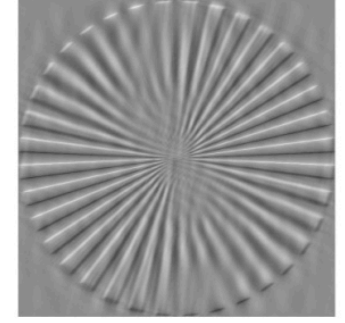

$X=-1.6 \mu \mathrm{m} \quad Y=-0.3 \mu \mathrm{m}$

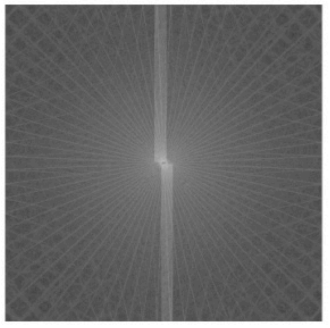

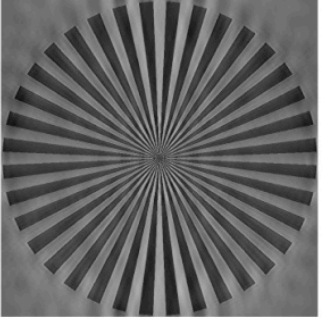

$X=0 \mu \mathrm{m} \quad \mathrm{Y}=-0.3 \mu \mathrm{m}$

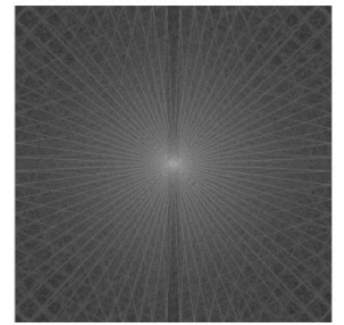

